Methods We identified FN patients admitted to in 2016. We implemented the proforma at the start of 2017. We subsequently collected data on FN patients admitted in 2017. We reviewed clinical records to compare their management in 2016 versus 2017 including length of stay (LOS) and time to antibiotics (TTA).

Results There were 33 oncology patients with 20 FN admissions in 2016. Antibiotics were given within 1 hour for $45 \%$ and the average TTA was 72 minutes. In 2017 there were 48 oncology patients with $42 \mathrm{FN}$ admissions. $17 \%$ of the admissions did not use the proforma. Antibiotics were given within 1 hour for $86 \%$ and the average TTA was 54 minutes. Risk stratification was completed for 45\% in 2017: 17\% were low risk and $28 \%$ were standard risk. $80 \%$ of the low risk patients were not discharged at 48 hours despite national recommendations. Average LOS was 4.2 days in 2016 and 5 days in 2017.

Conclusion The implementation of the FN proforma lead to improved standard of care in TTA of 54 minutes in comparison with national standard of 60 minutes mandated by the National Chemotherapy Advisory Group (NCAG). There has not been an identifiable improvement in the LOS, this suggests need for improving the understanding of the risk stratification and boosting the confidence in discharging low risk patients early. There is therefore scope for improvement in education and uptake of the risk stratification system to reduce LOS for FN patients.

\section{G183(P) CLINICAL MANAGEMENT OF ACUTE PAIN IN PAEDIATRIC SICKLE CELL DISEASE (SCD) FOLLOWING THE NICE GUIDANCE (CG143)}

${ }^{1} \mathrm{~L}$ Huckerby, ${ }^{1} \mathrm{R}$ Clubb, ${ }^{1,2} \mathrm{~A}$ Leigh. ${ }^{1}$ Paediatrics, Barts Health NHS Trust, London, UK;

2Paediatrics, University College Hospital, London, UK

\subsection{6/archdischild-2020-rcpch. 154}

Aims

- To assess if children with SCD presenting to our District General Hospital with an acute painful episode are managed in line with NICE guidance.

- To compare our results with previous audits (2014 and 2016).

- To use feedback from the multi-disciplinary team (MDT) to develop sustainable interventions to ensure guidance is met.

Methods We performed a retrospective case note review $(n=28)$ of medical notes. Primary data were collected from June-December 2018 and included all paediatric patients with SCD who presented in pain to the emergency department.

Results Our findings were presented to the MDT. Our local guideline was amended, with particular focus on the need to prescribe laxatives, anti-emetics and anti-pruritics to patients taking regular opioids, as well as timely pain relief. Feedback from the MDT, and reviewing interventions used by previous audits led us to amend induction for new doctors, emphasising the guideline and key audit findings.

Conclusions Clear improvements have been made from previous audits, however, despite this, there was a stark drop in our rates for offering analgesia within 30 minutes. This may reflect the challenges we face in providing rapid interventions in an ever-growing busy emergency department. Studies show

\section{Abstract G183(P) Table 1}

\begin{tabular}{llll}
\hline & 2018 & 2016 & 2014 \\
\hline Pain assessed on presentation using age appropriate score & $89 \%$ & $94 \%$ & $57 \%$ \\
Analgesia offered within 30 minutes of presentation & $32 \%$ & $65 \%$ & $60 \%$ \\
Nursing observations measured at presentation & $89 \%$ & $56 \%$ & $25 \%$ \\
Bolus of strong opioid offered in moderate-severe pain & $90 \%$ & $87 \%$ & $32 \%$ \\
Effectiveness of pain relief assessed every 30 minutes until a & $46 \%$ & $6 \%$ & $24 \%$ \\
satisfactory pain score was achieved & & & \\
Pain score reassessed every 4 hours & $91 \%$ & $13 \%$ & $38 \%$ \\
Laxatives offered to patients on opioids & $73 \%$ & $13 \%$ & $84 \%$ \\
Anti-pruritics and anti-emetics offered to patients on opioids & $46 \%$ & - & - \\
\hline
\end{tabular}

that specialist care is superior over generalist care and improves outcomes (Bundy et al. 2016; Evensen et al. 2016). The Sickle Cell Society suggests a Specialist Haemoglobinopathy Team with links to local hospitals could support acute pain services, as well as an individualised care plan for each patient.

This audit reinforces the importance of good acute care pathways and consideration should be given to alternative models of emergency care delivery.

\section{G184(P) DIAGNOSTIC DILEMMA IN A CHILD WITH BLEEDING}

AR Moosa, S Kapoor, R Gooding. Department of Paediatrics, University Hospitals of Leicester NHS Trust, Leicester, UK

\subsection{6/archdischild-2020-rcpch.155}

Introduction Acquired Thrombotic Thrombocytopenic Purpura (TTP) is a rare and severe disease in paediatric population. The known classic pentad of symptoms (microangiopathic hemolytic anemia, thrombocytopenia, acute kidney injury, fever and neurological symptoms) appear to be more varied rather than classical, in children. Herein, we describe a rare presentation of acquired TTP in a thirteen-year-old girl.

Case Report A thirteen-year-old girl previously fit and well, presented with a history of heavy vaginal bleeding, vomiting with increasing petechial rash over lower extremities and bruising to the chest wall. On presentation, she was febrile with generalised bruising. No history of trauma or significant past history nor family history to note.

Initial investigations showed anaemia and thrombocytopaenia (Haemoglobin of $63 \mathrm{G} / \mathrm{L}$, Platelet of $70 \times 10^{9} / \mathrm{L}$ ), raised Urea of $9 \mathrm{mmol} / \mathrm{L}$ and Lactate dehydrogenase $1292 \mathrm{IU} / \mathrm{L}$. Liver function and coagulation screen was normal. Blood film showed evidence of microangiopathic hemolytic anaemia (anisocytosis, schistocytes, spherocytes and basophilic stippling).

Differential diagnosis of Hemolytic Uraemic Syndrome (HUS) and TTP was considered at first instance.

She was initially stabilized in Paediatric Emergency Department where she received tranexamic acid infusion along with one unit of platelet and one unit of packed red cells.

In view of blood results and blood film, Haematology team considered TTP to be the likely diagnosis, henceforth she was transferred to tertiary renal unit for total plasma exchange (TPE). In addition, she received systemic steroids and immunosuppressant (Rituximab). 